\title{
Structural and Mechanical Properties of Thin Films of Bovine Submaxillary Mucin (BSM) vs. Porcine Gastric Mucin (PGM) on a Hydrophobic Surface in Aqueous Solutions
}

\section{Electronic Supplementary Information}

Jan Busk Madsen, ${ }^{1}$ Javier Sotres, ${ }^{2}$ Kirsi I. Pakkanen, ${ }^{1}$ Petr Efler, ${ }^{1}$ Birte Svensson, ${ }^{3}$ Maher Abou Hachem, ${ }^{3}$ Thomas Arnebrant, ${ }^{2}$ and Seunghwan Lee ${ }^{1}$

${ }^{1}$ Department of Mechanical Engineering, Technical University of Denmark

${ }^{2}$ Department of Biomedical Sciences, Faculty of Health and Society, Malmö University, 20506, Malmö, Sweden; Biofilms-Research Center for Biointerfaces, Malmö University, 20506 Malmö, Sweden.

${ }^{3}$ Enzyme and Protein Chemistry, Department of Systems Biology, Technical University of Denmark 


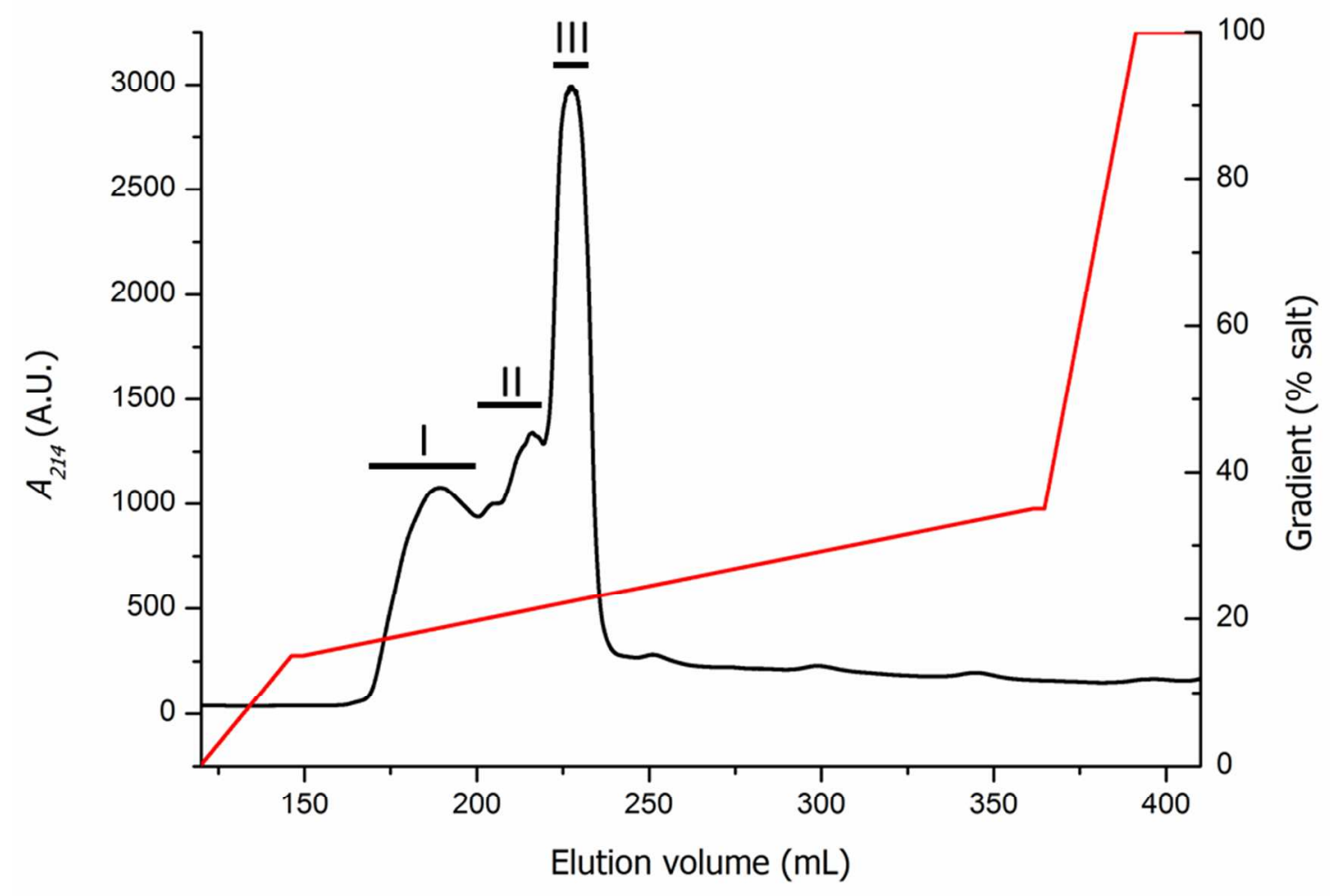

Figure S1. Chromatogram of the elution profile of PGM with the salt gradient indicated (red). The bars indicate analyzed regions in Figure S2. Part I, II, and III. 


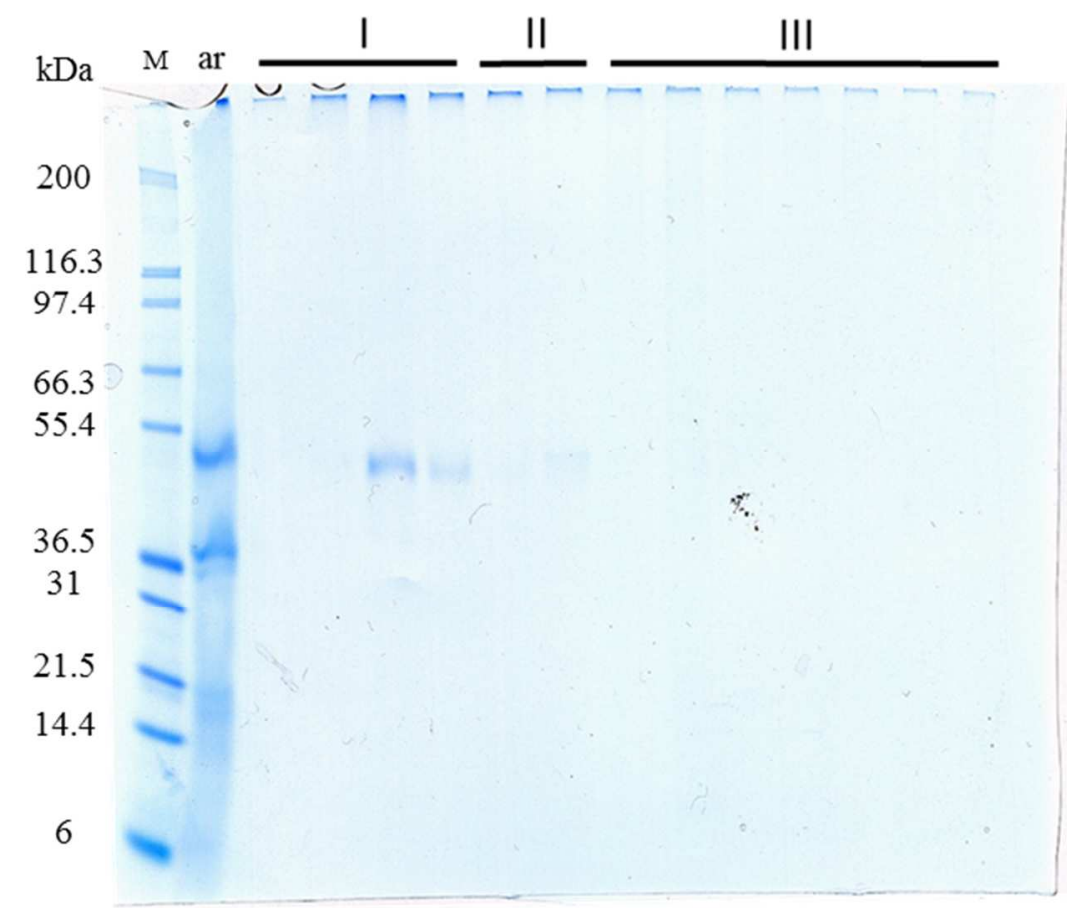

Figure S2. CBB stained SDS-PAGE analysis of fractions collected during anion exchange chromatography purification. The bars indicated I, II and III show fractions from the corresponding peaks in Figure S1. Peak III was selected for downstream applications. 


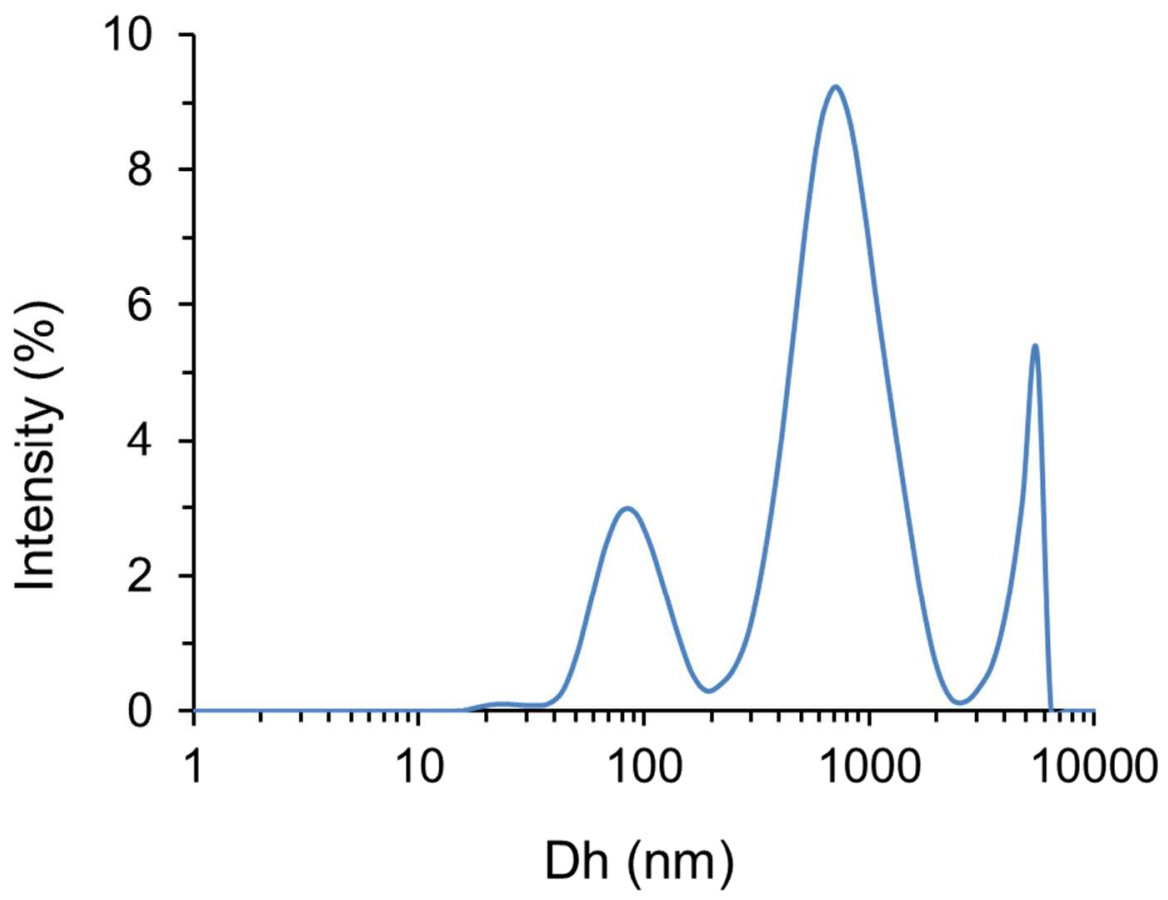

Figure S3. DLS measurements of arPGM at $\mathrm{pH}$ 7.4. The corresponding data for arBSM can be found in the reference 19 . 